\title{
Advantages and Functions of Clinical and Decision Support Systems
}

\author{
Dowse R \\ Pharmacy Division, University of Ghana, Legon Boundary, Accra, Ghana. \\ rdowseghana@gmail.com
}

\author{
Article Info \\ Doi: https://doi.org/10.53759/0088/JBSHA202202006 \\ Received 31 January 2021; Revised form 25 March 2021; Accepted 30 July 2021. \\ Available online 05 January 2022. \\ (C)2022 Published by AnaPub Publications.
}

Journal of Biomedical and Sustainable Healthcare Applications (http://anapub.co.ke/journals/jbsha/jbsha.html)

\begin{abstract}
Clinical Decision Support Systems (CDSSs) signify the framework shift in the medical sector in the modern age. CDSSs are utilized in augmenting healthcare facilities in the process of making complex clinical decisions. Since the first application of CDSSs in the 80s, the framework has witnessed significant transformation. The frameworks are now administered through electronic healthcare records with complex capacities. Irrespective of these complex advancements, there are existing questions concerning the impacts of CDSSs on service providers, healthcare costs, and patients' records. There are many published texts concerning the success stories of CDSSs, but significant setbacks have proved that CDSSs are not without any potential risks. In this research, we provide critical analysis on the application of CDSSs in clinical setting, integrating various forms, present application cases with proven effectiveness, potential harms and common pitfalls. We therefore conclude with evidence-centered recommendation for mitigating the issues of CDSSs maintainability, evaluation, implementation and designing.
\end{abstract}

Keywords - Diagnostic Decision Support System (DDSS), Clinical Decision Support Systems (CDSSs), Electronic Health Records (EHRs), Drug-Drug Integration (DDI).

\section{INTRODUCTION}

A Clinical Decision Support System (CDSS) [1] aims to enhance medical provision by supplementing clinical choices with specific medical skills, individual data, and other healthcare data. A conventional CDSS consists of application established to be an immediate assistance to clinical decision production whereby the independent patients' characteristics are paired to an automated clinical level of knowledge, and patient-specific guidelines or evaluations then presented to physicians for the decision-making process. CDSSs are widely applied at the point-of-care presently, permitting medical practitioners to incorporate their professionalism with recommendations or data provided by CDSSs. Nonetheless, CDSSs are widely being established that can utilize figures and facts, which could otherwise be unintelligible or unavailable to individuals. Computerized CDSSs have been around since the 1970s. They were time consuming, had poor network connectivity, and were generally restricted to academic interests at the time. There were also legal and ethical concerns expressed about the use of technology in healthcare, practitioner freedom, and who was to blame if a system with poor "interpretability" made a suggestion. Web-based connections and applications with Computerized Provider Order Entry (CPOE) [2] and Electronic Health Records (EHRs) [3] advancements are typically employed by CDSSs presently. They may be delivered through desktop, tablet, and smartphone, as well as biological surveillance and personal health technologies.

These technologies may or not provide outputs or be connected to EHR databases. Six CDSSs have been categorized and segmented into numerous groups and kinds, based on interventions time and if they are delivered actively or passively. Knowledge-based and non-knowledge-based CDSS are often used to classify CDSS. Guidelines ("IF" "THEN" expression) are established in the knowledge-centred networks, with systems receiving data to evaluate the protocols and providing output data and operations. Non-knowledge-centred CDSSs still require data sources, but instead of being planned to follow clinical expertise, the decisions are founded on Machine Learning (ML) [4], Artificial Intelligence (AI), or pattern recognition. Although non-knowledge-centred CDSSs are promptly diversifying application circumstances for $\mathrm{AI}$ in the clinical setting, they are fraught with potential challenges, e.g. lack of knowledge of reasoning utilized by AI to present black boxes and data accessibility difficulties. Elements of both types of CDSSs are similar, but vary, as illustrated in Fig. 1; and are established up of:

1. Foundation - the rules that have been coded into computer-based (knowledge0based), the approach, which is utilized to stimulate non-knowledge dependent decisions, and data that is accessible;

2. Inferential motor - Relates regulated or AI-determined guidelines and information structures to the individual 's medical data to generate an action and outcome, which is presented to the end users (for example, physician) through the;

3. Communication platform - Web pages, applications or EHRs frontend interactions via the end users engagements with the system. 


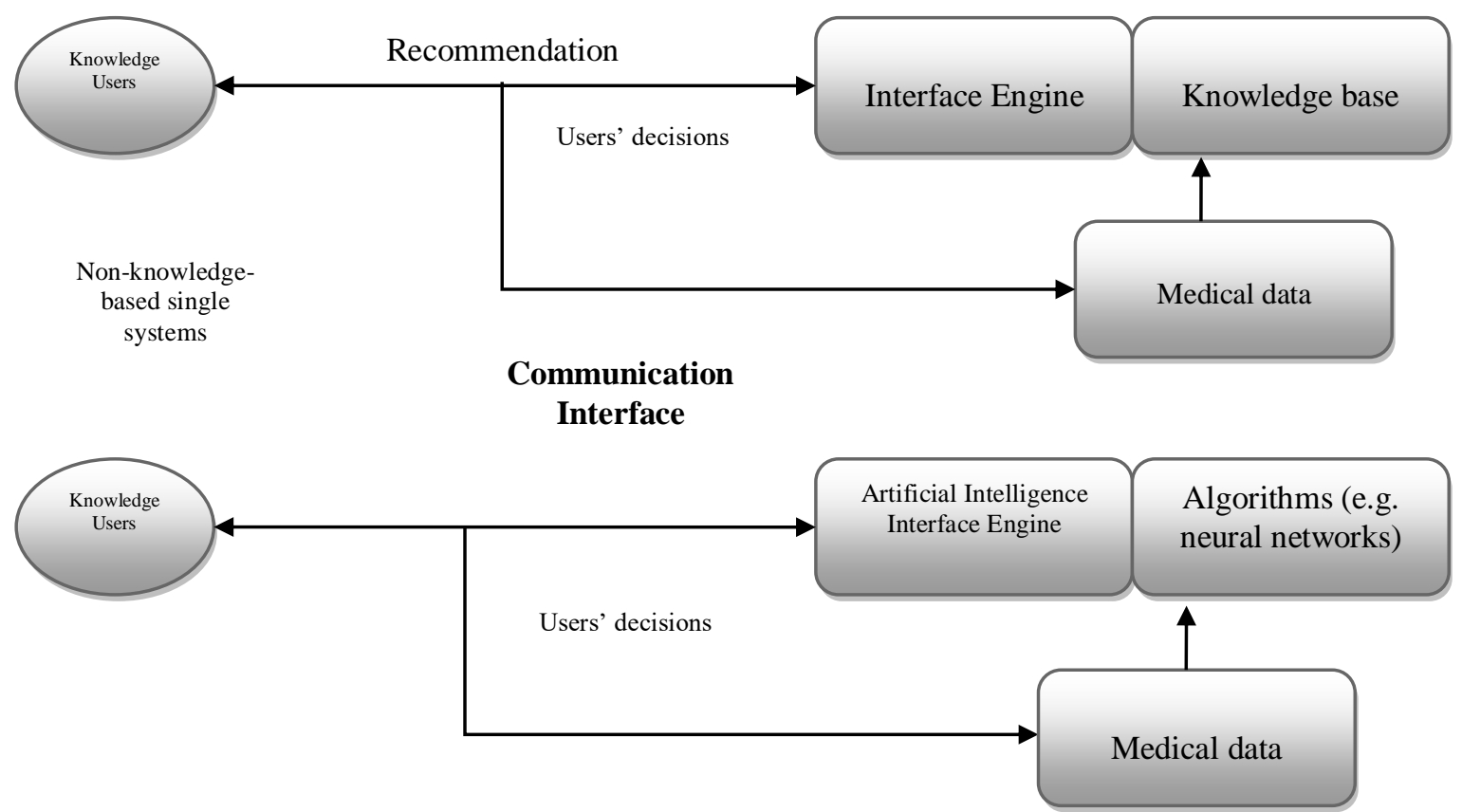

Fig 1. Major interactions in knowledge-centred and non-knowledge-centred CDSSs

The U.S. Government's Healthcare and Medicare Act have acknowledged CDSSs, pushing CDSS integrations into EHRs with fiscal incentives. In 2013, $41.1 \%$ of the U.S. facilities with EHRs also have CDSSs, whereas $40.2 \%$ of U.S., facilities has developed CDSS capacities in 2017. In some nations, EHRs' levels of acceptance have been promising with approximately $62 \%$ of medical practitioners in Canada utilizing them in 2013. The government of Canada, as well as Infoway, a non-profit organization, have given their support to the project. With up to 20 billion euros spent in medical Information Technology (IT) in 2010, England was also a global leader. Many nations, like Denmark, Estonia, Australia, and others, have managed in implementing national healthcare data, at least for patient-facing information.

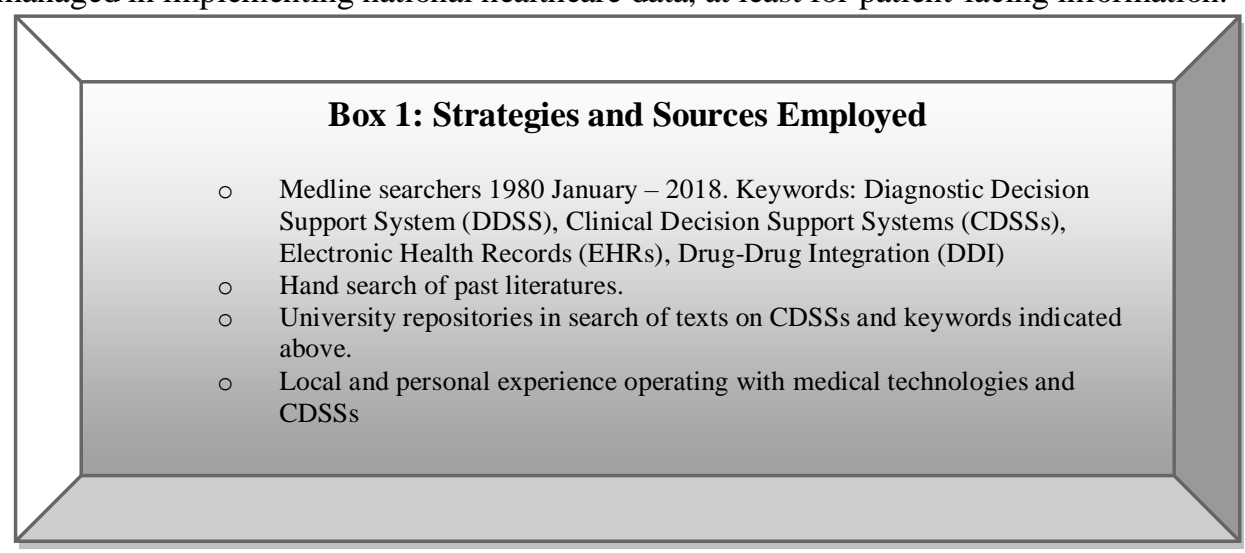

Fig 2. Strategies and sources employed in the research

The functional scope generated by CDSSs is massive, integrating wide-range diagnostics, alert systems, disease management schemes, prescriptions, and medicine control among others. They can potentially manifest any computerized reminders and alerts, computerized order sets, guidelines, patients' records, clinical workflows, and documentation templates. Every CDSSs functions will be illustrated in details in this paper. With the potentials and benefits of functionalities, including unplanned negative challenges, and strategies to mitigate potential challenges from CDSSs. The strategy utilized to inform reviews is presented in Box 1, Fig 2. Section II below presents an analysis of the advantages and functionality of CDSSs. Section III conclude the research.

\section{Patients' Safety}

\section{CDSS ADVANTAGES AND FUNCTIONS}

CDSSs is typically utilized in the efforts to minimize medical errors (see Table 1). Drug-Drug Integration (DDI) issues are prevailing and avoidable, with up to $65 \%$ of hospital patients subjected to one or more potential hazardous pairings. Drug security technologies for dosage duplicate of medicines, and DDI verification is increasingly included in CPOE platforms. These structures' notifications are one of the most widely used types of strategic planning. Nevertheless, researchers in [5] have discovered numerous variations concerning how DDI alerts are presents (such as disruptive/active or passive), 
techniques and prioritized utilized to identified DDIs. There are no protocols concerning the application of alerts to distributors, and the advancements typically indicate several dimensions of unnecessary alerts. In the U.S., the National Coordinator of Healthcare Information Technology has established the high-priority DDI list for CDSSs that has been approved and applied in CDSSs in other countries, e.g. Korea, Belgium and United Kingdom.

Table 1. Advantages, functions, challenges and remedies of CDSSs

\begin{tabular}{ll}
\hline $\begin{array}{c}\text { Advantages and functions } \\
\text { of CDSSs }\end{array}$ & Challenges of CDSSs \\
\hline $\begin{array}{l}\text { Patients' safety: } \\
\text { Minimizing the instances } \\
\text { of prescriptions and }\end{array}$ & $\begin{array}{l}\text { Alerts fatigue: } \\
\text { The phenomenon where } \\
\text { medication errors and }\end{array}$ \\
adverse occurrences. & $\begin{array}{l}\text { CDSSs recommendations } \\
\text { are available and } \\
\text { providers begin to dismiss } \\
\text { them irrespective of their } \\
\text { significance. }\end{array}$ \\
\end{tabular}

Medical management Following medical protocols and diagnostic reminders among others. users' knowledge

One instance is based on, or excessive trusts in the

\section{Remedies in mitigating the challenges \\ Details to the remedies}

Prioritizing
fundamental alerts,
reducing the
application of
disruptive alerts for
insignificant
indicators.

\section{Negative implication on Eliminate} prescriptiveness in accuracies of the framework. the designing of the system.

Assess the implication on the prevailing basis. specialties and personalization. designed to be specific.

Alerts fatigue could be thwarted by selecting and prioritizing alerts, which are fundamental, that will have a significant implication, and by designing alerts to particular DDI testing program has to be designed with algorithms, which integrates concomitant medications, laboratory values, patients' locations and administration timeframe, which is

Systems have to be structured in the aid of practitioners, without affective autonomy or being significantly definitive and prescriptive. It is fundamental to undertake an evaluation to visualize how systems are being utilized in the long run, after application. If accuracies are a problem, design transformations might have to be considered to prompt extra checking or order confirmation.

Management of costs
Minimizing the tests and
duplication of orders,
recommending affordable
treatment or medication
options, automated
tedious phases to
minimize the providers'
workloads.

\section{Fiscal issues}

Set-up could be costly (human resources or capital), and the longterm cost-efficacy is not assured

Designing and
planning for
longitudinal analysis
of costs at outlets.
Specifying the
measurements for
non-fiscal benefits
whenever applicable.
whenever applicable.
Administrative functions and automations

Diagnostic code selection, note auto-fill, and automatic documentations

\section{Systems and content Knowledge} maintainability issues

As practice transforms, there could be issues maintaining the content and knowledge protocols, which energize CDSSs to the present.

translations

\begin{tabular}{|c|c|}
\hline $\begin{array}{l}\text { Management } \\
\text { services in } \\
\text { with } \\
\text { concentration o } \\
\text { translations } \\
\text { CDSSs system } \\
\text { Measurement } \\
\text { systems } \\
\text { evaluation of } \\
\text { performance } \\
\text { CDSSs. }\end{array}$ & $\begin{array}{c}(\mathrm{KM}) \\
\text { place } \\
\text { major } \\
\text { on the }\end{array}$ \\
\hline
\end{tabular}

Management

concentration on the

of

ining individuals on why particular data entries and standardizations of information entry practices. Standards of planning KM have been presented.

It is fundamental to identify the transformations in the application and performance overtime. Moreover, the quality of the data repositories have to be traced, and it is fundamental to 


Diagnosis support
Issuing diagnostics
recommendations
centered on patients
records automating data
output from the results of
tests.

Users distrust of CDSSs

Individuals may disagree with the protocols given by CDSSs.

Reference

professional

knowledge integrates scientific reference in texts that are appropriate.

make sure that the conclusions are not being established on poor quality and corrupted datasets in prior.

To issue verifiable data sources to users concerning why recommendations are prevailing. Moreover, to enhance the degree of trust, this could provide more directions for individuals to upgrade their skillset in case they are unaware of recommendations.

Various systems also query the potentials for not pursuing recommendations to elucidate the mistrust source. This is the best idea; however, it must not be bulky or mandatory in designing.

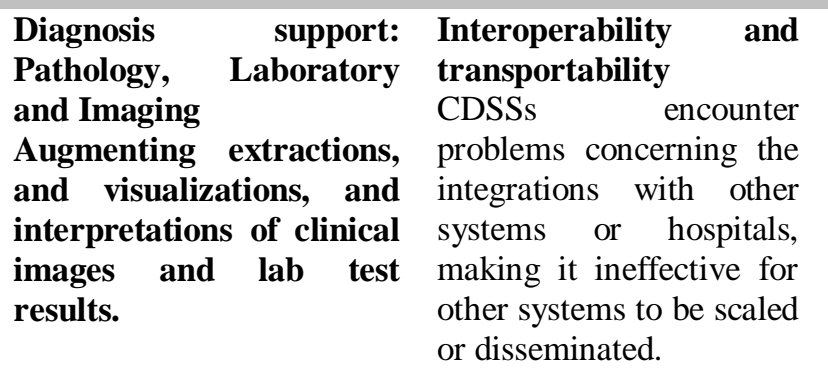
Implementation of Most open protocols for semantic and industrial standards structural interoperability and Securing blockchains and exchanges are being improved and cloud services. DICOM (Digital Imaging and Communication), SNOMED International, HL-7 (Hospital Level-7) for imaging protocols. Possibly, the protocols have to be applied in various dimensions within the medical sector, and with the external systems being utilized. Cloud-centered EHRs systems have to be significantly open architectures, and more flexible connections established between these systems. As for any clinical system, the aspect of security needs to be guaranteed through the basis of compliance with legislation eg HIPAA (Heath Insurance Portability and Accountability) in America, GDPR (General Data Protection Regulation) in Europe, and PIPEDA (Personal Information Protection and Electronic Documents Act) in Canada. In the upcoming decades, we may also visualize blockchain utilized to enable great interoperability and enhance security for HIE (Health Information Exchange).

$\begin{aligned} & \text { Patients' decisions } \\ & \text { support } \\ & \text { Decisions } \\ & \text { administered }\end{aligned} \begin{array}{r}\text { support } \\ \text { to the }\end{array}$
patients via individual
healthcare records,
including other applicable
systems.

\section{Dependencies on digital} literacy

CDSSs might necessitate high technological proficiencies to use.

\section{Conforming prevailing}

functionalities

Enough training conducted at launch. to Establishing consistencies with the interface of users of the existing systems. If there is one, it is fundamental to ensure the users do not have steeper learning curves to utilize systems.

Effective training modules have to be present for users. Training is planned in person by the medical leaders with vast EHRs experiences for generating buy-ins. Training should to present on the progressive basis, as novel staff 


\begin{tabular}{|c|c|c|c|}
\hline & & & $\begin{array}{l}\text { and individuals joining. One } \\
\text { methodology is to have onsite group } \\
\text { members designated as an elite user, } \\
\text { and able to provide sessions of } \\
\text { training. }\end{array}$ \\
\hline Effective documentation & $\begin{array}{l}\text { Poor quality and } \\
\text { inaccurate } \\
\text { documentation and data } \\
\text { CDSSs might aggregate } \\
\text { datasets from wide-range } \\
\text { sources, which are not } \\
\text { synced effectively. } \\
\text { Individuals could create } \\
\text { manuals, which } \\
\text { compromise } \\
\text { documentations and data. }\end{array}$ & $\begin{array}{l}\text { Professional } \\
\text { knowledge of } \\
\text { integrated systems } \\
\begin{array}{l}\text { Debugging and } \\
\text { testing during the } \\
\text { stages } \\
\text { development and } \\
\text { application. }\end{array}\end{array}$ & $\begin{array}{l}\text { The group has to be familiarized and } \\
\text { have professional knowledge of } \\
\text { external systems feeding data into } \\
\text { databases utilized by CDSSs. } \\
\text { Professional suggests testing medical } \\
\text { protocols for NPV and PPV during the } \\
\text { procedure of development and } \\
\text { application. In case users generate } \\
\text { datasets is a problem, it might be that } \\
\text { practitioners have not obtained } \\
\text { effective training on reading, } \\
\text { interpreting and responding to alerts, } \\
\text { or are dependent on clinicians for } \\
\text { checking clinical orders before the } \\
\text { process of dispensation. }\end{array}$ \\
\hline $\begin{array}{l}\text { Workflow advancement } \\
\text { CDSSs can expedite and } \\
\text { advance prevailing } \\
\text { medical workflows in } \\
\text { EHRs with effective } \\
\text { retrieval and } \\
\text { presentations of datasets. }\end{array}$ & $\begin{array}{l}\text { Fragmentation and } \\
\text { disrupted workflows } \\
\text { CDSSs can disrupt } \\
\text { prevailing workflows in } \\
\text { case they need } \\
\text { interactions externals to } \\
\text { EHRs, and do not match } \\
\text { the giver's actual world } \\
\text { data processing sequence. }\end{array}$ & $\begin{array}{l}\text { Usage assessment } \\
\text { Workflows models. }\end{array}$ & $\begin{array}{l}\text { Iterative and rigorous usability } \\
\text { assessments and pilot tests have to be } \\
\text { done on CDSSs before utilizing them } \\
\text { in the medical setting. Various } \\
\text { applicability evaluation tools are } \\
\text { present, alongside quantitative } \\
\text { approaches and models. } \\
\text { Unless CDSSs goals is to transform } \\
\text { the care procedure, CDSSs have to be } \\
\text { create to fit or conform within the } \\
\text { present users' workflows }\end{array}$ \\
\hline
\end{tabular}

The Barcode Point-of-Care (BPOC) and Electronic Drug Dispensing System (EDDS) drug administering methods are two more methods that are aimed at improving safety of patients. Each stage of the procedure (writing prescriptions, translating, administering, and delivering) is automated and takes place inside a networked system. At the time of administering, the drug is immediately detected using barcodes or Radio-Frequency Identification (RFID) the patients' data and instructions are cross-checked. Another possible advantage of CDSS is the reduction of medicine delivery mistakes at the 'bedside' (opposed to further upstream). Because of the high technological demands and prices, adoption is rather modest. These methods are effective at decreasing mistakes. Most of these technologies may be used in conjunction with CPOE and CDSS, resulting in lower prescription failure rates for medication allergy diagnosis, inappropriate dose, and incorrect or ambiguous orders, according to [6]. Providers that ignore or purposefully operate around the technology, like with most CDSS, might still make mistakes.

In addition to medication-related cardiac events, CDSS improves patients' security by providing notification mechanisms for additional medical occurrences. A CDSS for plasma glucose monitoring in the intensive care unit, for instance, was capable of minimizing the frequencies of hypo-glycemic cases. CDSSs motivated healthcare practitioners to retrieve glycogen samples centred on the localized surveillance system protocols that stated how frequently measure have to be considered with respect to personal features and previous glucose level classes. Generally, CDSSs purposed to enhance the safety of patients through CPOE and advancements have been efficient in minimizing prescriptions, and dosage mistakes, incompatibilities through computerized warnings, drug-event surveillance, and other problems. The case of patients is a secondary objective (or prerequisite) of practically every CDSS, regardless of their main goal.

\section{Medical Management}

CDSSs have been identified in [7] to enhance compliance to clinical recommendations. Standardized clinical recommendations and diagnostics pathway have been shown to be problematic in the process of applying in practice because of poor adherence by physicians. The expectations that clinicians would read, comprehend and implement novel protocols have not proved to be accurate. The protocols implicitly integrated in recommendation, on the contrary, could be physically recorded within CDSSs. CDSSs could assume various forms, integrating the standardized practice protocols for particular conditions, notifications to certain procedures for the users engaged, testing the reminders etc. CDSSs might also aid with the management of patients on assessing protocols, surveillance, and making potential prescriptions, referral follow-ups, and guaranteeing preventive Medicare. CDSSs might also notify clinicians whenever users could be follow-up with the treatment procedures, or are about to follow-up, and to assess the patients that are study candidates that depend on 
a particular factor. When a patient's information satisfies clinical research requirements, CDSSs established Cleveland clinic point-of-care notices to medical practitioners. The alert directs users to fill out forms, which confirm their qualifications and consent to contact patients, and send records to the research panel, and product record sheets for medical research.

\section{Management of Costs}

Medical intervention reducing the in-patient duration of stay, CPOE-oriented advancements recommending cheaper pharmaceutical alternatives, or eliminating test repetition may all help healthcare schemes save funds. In the paediatric cardiac ICU (Intensive Care Unit), the CPOE-protocol was created to confine the order to blood works, chemistry, and anti-coagulant screen to a specific interval (i.e. 24-hour). This amounted to $\$ 717,538.50$ yearly cost reduction by minimizing scientific capability consumption without adjusting LoS (Length of Stay) or fatalities. CDSSs could inform individuals of the affordable diagnostic situations and options, which insurance providers will handle. Various clinics in Germany are transformed to medications from clinical drug prescription. The Heidelberg Clinic established a drug switch methodology and applied it into their present CPOE platforms following the discovery that one of every five errors, advancing practitioners' security, minimizing costs, and minimizing efforts.

\section{Administrative Functions and Automations}

Diagnostic and medical coding, operations and test administrations, and patients' triage are endorsed by CDSSs. Developed methodologies could provide an enhanced list of medical data to aid practitioners in the process of selecting effective ones. CDSSs were established to overcome inconsistencies in ICD-9 admissions coding in EDs (Emergency Departments). To aid EDs experts identify diagnosis admission code promptly, technological featured anatomographical displays (visuals, interactive portrayals of nervous systems) interlinked to ICD codes. CDSSs could fundamentally boost the dependability of clinical records. When compared to control facilities, obstetrics CDSSs with expanded prompting systems dramatically enhanced justification recordings for induction of labor and estimated fetal weights. The recording quality is fundamental because it could directly enhance medical procedures. For example, CDSSs were introduced to guarantee that individuals were appropriately vaccinated after splenectomy, in order to prevent the higher risk of illness (such as pneumonia, Haemophilus influenzae, meningococcal, and other illnesses) associated with splenectomy. The authors discovered, nevertheless, that $71 \%$ of individuals possessing the phrase "splenectomy" in EHRs did not have it quoted in the issue list (that is what stimulates CDSSs alerts). Afterwards, supplementary CDSSs was established to enhance the applicability of initial vaccine CDSSs by enhancing the problem list records of splenectomy.

\section{Diagnostics Support}

Diagnostic Decision Support System (DDSS) are utilized for diagnosis approaches. The systems have typically integrated computational diagnosis or screening phase, whereby they receive datasets and users' selections, and then generate lists of plausible or probable diagnosis. Nonetheless, DDSS has not have similar implication on other types of CDSSs owing to wide-range factors, integrating unfavourable medical prejudices and attitudes, lower precision (frequently because of dataset gaps), and inefficient systems' engineering that requires human data input. Better EHR connection and standard language, such as Snomed Medical Terms, are helping to improve the latter.

Authors in [8] developed a DDSS for the assessment of diabetic neuropathy using fuzzy approach, which is a nice sample of a successful DDSS. They obtained 93\% accuracy in detecting motors, perceptual, combined neuropathies, and usual cases using data input that included indications and medical test results when contrasted to specialists. While this is very useful, particularly in places where established diagnostic specialists are few, also there is a need for technologies that can augment professional diagnosis. DXplain is a computerized reference-based DDSS that offers a prognosis based on medical symptoms. In a random controlled experiment including 87 family practice residents, which integrates the randomly allocated to use the system undertaken better on validated diagnostic examinations integrating 30 medical manifestations (84\% against $74 \%$ ). Provided the high rates of diagnosis errors, mostly in basis care, CDSSs and Information Technology (IT) options have much potential to diversify diagnostics. Non-knowledge-centred methodologies such as deep learning are presently being utilized to establish diagnostic algorithms that could pave way for more precise diagnostics. Babylonian AI-powered Triage and Diagnostics Networks in the UK is a fundamental illustration of promise, and work, which has to be conducted before these technologies are effective for primetime.

\section{Diagnosis Support}

Image centred on data CDSSs are typically utilize for the interpretation of images whereby they could aid clinicians select the most effective tests to perform, remind them regarding the comparison of restrictions. At Virginia Mason Medical Center, interventions CDSSs for image procurement was highlighted to fundamentally minimize the application of spine MRI for chronic systems in the back, head MRI for headache and sinus CR for sinus. Before image ordering, CDS necessitates sustainability. Fundamentally, in case an image has been neglected, algorithms provide an alternative. Another marketed framework is RadWise ${ }^{\circledR}$ that analyses patients' symptoms and matches them to a big database of diagnosis, as well as offering suitable usage suggestions at the time of treatment. Non-knowledge-centred CDSSs for effective imagery and precise radiomics (radiography) has attracted more attentions. 
Practitioners want technology to assist them in retrieving, displaying, and analyzing pictures, which account for a growing volume of health data yet require substantial human evaluation. AI is proving to be capable of producing insights that humans could not. These initiatives fulfil this by utilizing powerful pixel identification and image classification model, the most distinguished of which being DL (Deep Learning ). IBM Watson Healthcare, Google, DeepMind, and other entities are on the cutting edge, creating tools for disease diagnosis, diagnostic imaging interpretations, diabetes retinal diagnostics, Alzheimer's diagnosis using multimodal pattern recognition, and a slew of other applications. The 'Eyes of Watson' from IBM Watson has been able to integrate computer vision of a medical examination with script identification of case details to give full decision assistance (or what IBM refers to a cognitive aid).

Numerous initiatives have shown efficiency that is 'on par' with that of human specialists. For example, Google's analysts developed Complex Convolutional Neural Networks (CNN) to identify diabetes mellitus (vascular injury in the eye) with a sensitive and specific from a database of 130,000 retina pictures. The method's effectiveness was comparable to that of board-certified ophthalmologists in the United States. Another research released lately by the Stanford team proved that a CNN for identifying arrhythmias on an ECG outperformed the mean cardiologist's efficiency (F1 and sensitivities with matching specificity) across all rhythm categories. With the present pace of improvement, some specialists predict that in 15-20 decades, computers will be doing (or at least pre-processing) the bulk of diagnostic analysis. For the time being, nevertheless, we should consider these early technologies as a supplement to a clinician's existing toolkit.

\section{Diagnosis Support: Pathology, Laboratory and Imaging}

CDSSs could also aid with laboratory that is a sub-segment of diagnosis. EHRs platforms, reminders and notifications for aberrant test results are widespread and straightforward. CDSSs could also be utilized to prolong the significance of laboriented testing to eliminate intrusive or riskier diagnosis. Liver biopsies are the gold protocols for the treatment and diagnosis of Hepatitis B and Hepatitis C; however non-invasive laboratory methodologies are not precise enough to be approved. Nevertheless, AI algorithms that incorporate various examinations (serum indicators, imagery, and gene testing) are being designed to achieve substantially higher precision. CDSS may also be used as an interpretation aid when the comparison ranges of a sample are highly individualized, such as by age, gender, or disease subgroups. Several other clinical specialities rely on pathology results as decision-making tools. Some CDSS may be used to grade tumors automatically. This was done with up to $93 \%$ accuracy for classifying urinary bladder tumors and predicted renewal. The categorization and classifying of brain tumors has been performed in the same way. Automated ECG assessment, computerized arterial plasma gas interpretations, protein electrophoresis results, and CDSS for plasma cell counts are only a few illustrations.

\section{Patients' Decisions Support}

We are seeing CDS capabilities incorporated, comparable to EHRs, with the individual as the end customer or "director" of the information, thanks to the development of individual healthcare records. This is a fundamental phase towards patient-based diagnostics, and CDSS-supported EHRs are actually the perfect instrument for implementing shared decision-making among providers and the patients, especially since CDSS may eliminate a client's lack of knowledge as an obstacle to their own treatment. EHRs are typically created as add-ons to commercialized EHR systems or as stand-alone web-based or mobile apps. When linked to EHRs, Personal Health Records (PHRs) [9] could have a two-way interlinkage, whereby data submitted explicitly by individuals can be made available to their healthcare practitioners and datasets from EHRs for users to see. The interface of customers, one of the PHRs, was a dashboard allowing individuals to check prescriptions and test results as well as connect with their doctors.

This has progressed to the point where some networks now enable patients to make changes to their own medical records, affecting EHR information as well. Vanderbilt University's MyHealthAtVanderbilt, a PHR completely incorporated within the organizational EHR, is another model. They included a Flu Toolkit for individuals with flu-like signs to determine the amount of healthcare they need and then assist them in seeking care, in additional to genetic disorder distribution of patient educational resources. System surveillance [10] is an important and popular element of PHRs, but the data obtained is closely endless, fluctuating from sensitivities to healthcare insurance to prescriptions and drug data. In addition, PHRs and other patients' surveillance apps may be developed to gather data from health monitors and other sensors in order to give clinicians with relevant insights. In the field of diabetes care, there is a great illustration. Many technologies are currently in use, but one developed by Stanford Medical School employs a peripheral glucose monitor that sends information to an Apple iPhone (HealthKit). Apple established the HealthKit compatible with Epic's HER (Electronic Health Records) and "MyChart" PHRs. This allows practitioners to track personal glucose level among visits and access them through "MyChart" for follow-up and render prompt advice. A pilot research showed enhanced provider efficiency, patient engagement, and, eventually, quality care. 82 Cardiac arrest (cardiology), hypertensive, sleep problems, palliative/elder care, and other healthcare sectors are adopting comparable surveillance technologies that incorporate PHR/EHR, wearable technology, and CDSS. It is important to note that, as PHRs have progressed with CDSS abilities, more focus has been placed on the configuration of these processes to support participatory decision among patients and providers, as well as to be immersive tools that help clients become more competent and engaged in their individual diagnostic. PHRs, which act as a storehouse for healthcare data are significantly perceived as falling short, especially by individuals. 


\section{Effective documentation}

This includes enhancing quality and correcting inaccurate documentation and data. CDSSs might aggregate datasets from wide-range sources, which are not synced effectively. Individuals could create manuals, which compromise documentations and data. The group has to be familiarized and have professional knowledge of external systems feeding data into databases utilized by CDSSs. Professional suggests testing medical protocols for NPV and PPV during the procedure of development and application. In case users generate datasets is a problem, it might be that practitioners have not obtained effective training on reading, interpreting and responding to alerts, or are dependent on clinicians for checking clinical orders before the process of dispensation.

\section{Workflow advancement}

CDSSs can expedite and advance prevailing medical workflows in EHRs with effective retrieval and presentations of datasets. CDSSs can disrupt prevailing workflows in case they need interactions externals to EHRs, and do not match the giver's actual world data processing sequence. Iterative and rigorous usability assessments and pilot tests have to be done on CDSSs before utilizing them in the medical setting. Various applicability evaluation tools are present, alongside quantitative approaches and models. Unless CDSSs goals is to transform the care procedure, CDSSs have to be create to fit or conform within the present users' workflows

\section{CONCLUSION}

Clinical Decision Support Systems (CDSSs) have proved to integrate medical practitioners in wide-range decisions and patients' care tasks, and presently, they ubiquitously and actively support delivery schemes for quality Medicare. Most of the CDSSs application provides a basis of proof behind them, mostly those oriented to Computerized Provider Order Entry (CPOE). The provision of support for CDSSs proceeds to complement the era of Electronic Health Records (EHRs), and there are significantly sophisticated to be made integrating interoperability, ease-of-deployment, affordability and speed. Similarly, it is fundamental to remain cautious for probably downfall of CDSSs that range from typically not operating and vandalizing resources, to fatiguing care givers and affect the quality of Medicare. Therefore, more precautions and conscientious designing should be considered when developing, applying and sustaining CDSSs. A segment of these elements have been survey in this research; however, more work is needed, as CDSSs progresses in terms of complexity based on the advancement of Artificial Intelligence (AI), data analytics and interoperability.

\section{References}

[1]. R. B. Haynes, N. L. Wilczynski, and Computerized Clinical Decision Support System (CCDSS) Systematic Review Team, "Effects of computerized clinical decision support systems on practitioner performance and patient outcomes: methods of a decision-maker-researcher partnership systematic review," Implement. Sci., vol. 5, no. 1, p. 12, 2010.

[2]. M. Kinlay, W. Y. Zheng, R. Burke, I. Juraskova, R. Moles, and M. Baysari, "Medication errors related to computerized provider order entry systems in hospitals and how they change over time: A narrative review," Res. Social Adm. Pharm., vol. 17, no. 9, pp. 1546-1552, 2021.

[3]. I. V. Angel and Department of Health Informatics and Analytics, Tufts University, School of Medicine, MA, USA, "A physician's commentary on electronic health records in the United States medical practice," Public Health (Fairfax), vol. 6, no. 1, pp. 9-11, 2021.

[4]. S. S. Gornale, S. Kumar, and P. S. Hiremath, "Handwritten signature Biometric data analysis for personality prediction system using machine learning techniques," Trans. mach. learn. artif. intell., vol. 9, no. 5, pp. 1-22, 2021.

[5]. A. Bellesoeur et al., "Characterizing the risk of drug-drug interactions in sarcoma treated patients: Role of pharmacist integration," Ann. Oncol., vol. 29 Suppl 8, no. suppl_8,pp. viii753-viii754, 2018.

[6]. K. Grunnet and J. E. Turrentine, "Patch testing in the diagnosis of medication allergy," Curr. Treat. Options Allergy, vol. 3, no. 3, pp. 310321, 2016.

[7]. S. Crepin et al., "Compliance of French academic clinical trials with the Clinical Trial Facilitation and Coordination Group recommendations on contraception and pregnancy testing requirements," Clin. Trials, vol. 17, no. 3, pp. 314-322, 2020.

[8]. F. Philp, A. Faux-Nightingale, S. Woolley, E. de Quincey, and A. Pandyan, "Implications for the design of a Diagnostic Decision Support System (DDSS) to reduce time and cost to diagnosis in paediatric shoulder instability," BMC Med. Inform. Decis. Mak., vol. 21, no. 1, p. 78, 2021 .

[9]. S. Boch, E. Sezgin, D. Ruch, K. Kelleher, D. Chisolm, and S. Lin, "Unjust: the health records of youth with personal/family justice involvement in a large pediatric health system," Health Justice, vol. 9, no. 1, p. 20, 2021

[10]. National Nosocomial Infections Surveillance System, "National Nosocomial Infections Surveillance (NNIS) System Report, data summary from January 1992 through June 2004, issued October 2004,” Am. J. Infect. Control, vol. 32, no. 8, pp. 470-485, 2004. 